\title{
Chrysanthemum-like high-entropy diboride nanoflowers: A new class of high-entropy nanomaterials
}

\author{
Da LIU, Honghua LIU, Shanshan NING, Yanhui CHU* \\ School of Materials Science and Engineering, South China University of Technology, Guangzhou 510641, China
}

Received: February 26, 2020; Accepted: March 8, 2020

(C) The Author(s) 2020.

\begin{abstract}
High-entropy nanomaterials have been arousing considerable interest in recent years due to their huge composition space, unique microstructure, and adjustable properties. Previous studies focused mainly on high-entropy nanoparticles, while other high-entropy nanomaterials were rarely reported. Herein, we reported a new class of high-entropy nanomaterials, namely $\left(\mathrm{Ta}_{0.2} \mathrm{Nb}_{0.2} \mathrm{Ti}_{0.2} \mathrm{~W}_{0.2} \mathrm{Mo}_{0.2}\right) \mathrm{B}_{2}$ high-entropy diboride (HEB-1) nanoflowers, for the first time. Formation possibility of HEB-1 was first theoretically analyzed from two aspects of lattice size difference and chemical reaction thermodynamics. We then successfully synthesized HEB-1 nanoflowers by a facile molten salt synthesis method at $1423 \mathrm{~K}$. The as-synthesized HEB-1 nanoflowers showed an interesting chrysanthemum-like morphology assembled from numerous well-aligned nanorods with diameters of $20-30 \mathrm{~nm}$ and lengths of $100-200 \mathrm{~nm}$. Meanwhile, these nanorods possessed a single-crystalline hexagonal structure of metal diborides and highly compositional uniformity from nanoscale to microscale. In addition, the formation of the as-synthesized HEB-1 nanoflowers could be well interpreted by a classical surface-controlled crystal growth theory. This work not only enriches the categories of high-entropy nanomaterials but also opens up a new research field on high-entropy diboride nanomaterials.
\end{abstract}

Keywords: high-entropy materials; diborides; nanomaterials; molten salt synthesis

\section{Introduction}

High-entropy materials, including high-entropy alloys and ceramics, that consist of four or more principal elements in near-equiatomic ratios or at least with each elements being between 5 and 35 at $\%$ in a single-phase structure have attracted significant attentions since 2004 owing to their huge composition space, unique microstructure, and adjustable properties [1,2]. In the past decade, the research of high-entropy materials

* Corresponding author.

E-mail: chuyh@scut.edu.cn mainly focused on high-entropy alloys. Extensive experimental studies have demonstrated that high-entropy alloys exhibit many impressive properties, such as superior strength and ductility, good corrosion resistance, and interesting creep characteristics [3-5]. Inspired by high-entropy alloy concept, the newly developed field of high-entropy ceramics has been gaining growing attention in recent years for potential applications in structural and functional fields, especially extreme conditions of temperature, pressure, and others. Up to date, numerous attempts have been made to exploring a variety of high-entropy ceramics, including metal oxides [2,6,7], carbides [8-12], and diborides [13-16] 
with many superior physical and chemical performances, such as high hardness, low thermal conductivity, good thermodynamics stability and corrosion resistance, superior electrochemical and physicochemical properties. Compared with their bulk counterparts, high-entropy nanomaterials, including high-entropy nanoparticles, nanorods/wires/tubes, nanoplates, nanoflowers, etc., have much more unique physical and chemical characteristics due to their five core effects including small size effect, surface effect, quantum size effect, quantum tunneling effect, and dielectric confinement effect $[17,18]$. Therefore, the recent research has begun to focus on the synthesis of high-entropy nanomaterials. Nevertheless, only some high-entropy alloy and ceramic nanoparticles have been successfully explored until now [19-21]. Extending state of the art for new classes of high-entropy nanomaterials is still a great challenge for the scientific community.

High-entropy diborides, as a number of high-entropy ceramics, are very attractive owing to the unusual combination of their attractive physico-chemical properties for their diboride components, such as melting temperature exceeding $3000 \mathrm{~K}$, high hardness, chemical inertness, good electrical and thermal conductivity, low neutron absorption, etc. [13-16]. Meanwhile, compared with other nanostructures, the nanoflowers may possess much more unique physical and chemical characteristics due to their special and complex nanostructures [22]. Therefore, in this work, we open up new opportunities for the research of a new class of highentropy nanomaterials, namely $\left(\mathrm{Ta}_{0.2} \mathrm{Nb}_{0.2} \mathrm{Ti}_{0.2} \mathrm{~W}_{0.2} \mathrm{Mo}_{0.2}\right) \mathrm{B}_{2}$ high-entropy diboride (HEB-1) nanoflowers. We first analyzed the formation possibility of HEB-1 nanoflowers from two aspects of lattice size difference and chemical reaction thermodynamics and then successfully synthesized the chrysanthemum-like HEB-1 nanoflowers by a facile molten salt synthesis method at $1423 \mathrm{~K}$. Phase composition, microstructure, and compositional uniformity of the as-synthesized HEB-1 nanoflowers were investigated, as well as their growth mechanisms. To the best of our knowledge, it is the first time that high-entropy nanoflowers are synthesized and investigated. This work not only enriches the categories of high-entropy nanomaterials but also will explore a new research field in high-entropy diboride nanomaterials.

\section{Experimental procedure}

Commercially available $\mathrm{Nb}_{2} \mathrm{O}_{5}$ and $\mathrm{Ta}_{2} \mathrm{O}_{5}$ powders
(99.9\% purity, particle size 1-3 $\mu \mathrm{m}$, Shanghai ChaoWei Nanotechnology Co. Ltd., Shanghai, China), $\mathrm{TiO}_{2}$, $\mathrm{WO}_{3}$, and $\mathrm{MoO}_{3}$ powders $(99.9 \%$ purity, particle size 100-300 nm, Shanghai ChaoWei Nanotechnology Co. Ltd., Shanghai, China), and amorphous B powders (98.0\% purity, average particle size $<3 \mu \mathrm{m}$, Shanghai ChaoWei Nanotechnology Co. Ltd., Shanghai, China) were used as starting materials. $\mathrm{KCl}$ was used as molten salt medium. Details of the synthesis of HEB-1 nanoflowers were described as follows: the aforementioned starting materials and $\mathrm{KCl}$ salts were first milled by hand for $30 \mathrm{~min}$ in an agate mortal using an agate pastel based on following ratios: 1:1:2:2:2:35 for $\mathrm{Ta}_{2} \mathrm{O}_{5} / \mathrm{Nb}_{2} \mathrm{O}_{5} / \mathrm{TiO}_{2} / \mathrm{WO}_{3} / \mathrm{MoO}_{3} / \mathrm{B}$ (molar ratio), and 10:1 for $\mathrm{KCl} /\left(\mathrm{Ta}_{2} \mathrm{O}_{5}, \mathrm{Nb}_{2} \mathrm{O}_{5}, \mathrm{TiO}_{2}, \mathrm{WO}_{3}, \mathrm{MoO}_{3}, \mathrm{~B}\right)$ (weight ratio). The mixture of above powders was put into an alumina crucible and then placed into a horizontal alumina tube furnace. Afterwards, the furnace was heated at a rate of $10 \mathrm{~K} / \mathrm{min}$ to the desired temperature and then maintained for $30 \mathrm{~min}$. The whole heating and cooling process was carried out in flowing argon gas. After cooling naturally to room temperature, the as-synthesized products were taken out, immersed in the deionized water at $363 \mathrm{~K}$ and absolute ethanol at $273 \mathrm{~K}$, and filtered onto Nuclepore filters to remove the residual $\mathrm{KCl}$ salts and $\mathrm{B}_{2} \mathrm{O}_{3}$ products. Finally, they were repeatedly washed, filtered, and dried at $333 \mathrm{~K}$. In addition, to investigate the growth mechanism of HEB-1 nanoflowers, additional experiments were conducted at $1423 \mathrm{~K}$ for the different holding time (10, 20, and $30 \mathrm{~min})$.

Phase compositions of the as-synthesized products were analyzed by X-ray diffraction (XRD, X'pert PRO; PANalytical, Almelo, the Netherlands). Equivalent counting time for a conventional point detector would be $30 \mathrm{~s}$ per point at $0.01^{\circ} 2 \theta$ increment. Microstructure and compositional uniformity of the as-synthesized products were characterized by scanning electron microscopy (SEM, Supra-55; Zeiss, Oberkochen, Germany) equipped with energy dispersive spectroscopy (EDS) and transmission electron microscopy (TEM, Tecnai F30G2; FEI, Eindhoven, the Netherlands) equipped with EDS.

\section{Results and discussion}

To analyze the synthesis possibility of HEB-1 products by molten salt synthesis method, a critical empirical 
parameter, lattice size difference $(\delta)$, that is usually calculated to predict the formation ability of solid solutions is first analyzed and it can be expressed in high-entropy diborides by the following equation [23]:

$$
\delta=\sqrt{\sum_{i=1}^{n} \frac{n_{i}}{2}\left[\left(1-\frac{a_{i}}{\bar{a}}\right)^{2}+\left(1-\frac{c_{i}}{\bar{c}}\right)^{2}\right]}
$$

where $n$ is the metal diboride component species in high-entropy diborides, $n_{i}$ is the molar fraction of the $i$ th $\mathrm{MeB}_{2}$ component of high-entropy diborides. $a_{i}$ and $c_{i}$ are corresponding lattice parameters of individual metal diborides, and $\bar{a}=\sum_{i=1}^{n} n_{i} a_{i}$ and $\bar{c}=\sum_{i=1}^{n} n_{i} c_{i}$ are average lattice parameters, respectively. In general, a smaller $\delta$ indicates a smaller lattice size difference and finally benefits the formation of solid solutions. According to the reported lattice parameters of individual metal diborides [13,22], the $\delta$ value of HEB-1 can be calculated to be $2.763 \%$, less than that of the previously reported $\left(\mathrm{Hf}_{0.2} \mathrm{Zr}_{0.2} \mathrm{Ta}_{0.2} \mathrm{Nb}_{0.2} \mathrm{Ti}_{0.2}\right) \mathrm{B}_{2}$ high-entropy diborides (3.109\%) [13], which suggests that the HEB-1 product can be synthesized by molten salt synthesis method from the aspect of lattice size difference. In addition, the thermodynamics of the possible chemical reactions for the starting materials is further analyzed. In our work, the starting materials mainly consist of $\mathrm{TiO}_{2}, \mathrm{Nb}_{2} \mathrm{O}_{5}, \mathrm{Ta}_{2} \mathrm{O}_{5}, \mathrm{WO}_{3}, \mathrm{MoO}_{3}$, and $\mathrm{B}$ powders, while $\mathrm{KCl}$ salts are only utilized as molten salt medium. Therefore, on the basis of the reported thermodynamic data of individual metal diborides [24], the possible reactions as well as correlations between standard Gibbs free energy $\left(\Delta G_{R, T}^{\theta}\left(\mathrm{kJ} \cdot \mathrm{mol}^{-1}\right)\right)$ of these starting materials and temperature $(T(\mathrm{~K}))$ can be described as follows:

$$
\begin{aligned}
\mathrm{TiO}_{2}+\frac{10}{3} \mathrm{~B} & \rightarrow \mathrm{TiB}_{2}+\frac{2}{3} \mathrm{~B}_{2} \mathrm{O}_{3}(\mathrm{l}) \\
\Delta G_{R, T}^{\theta} & =-223930+4 T \\
\frac{1}{2} \mathrm{Nb}_{2} \mathrm{O}_{5}+\frac{11}{3} \mathrm{~B} & \rightarrow \mathrm{NbB}_{2}+\frac{5}{6} \mathrm{~B}_{2} \mathrm{O}_{3}(\mathrm{l}) \\
\Delta G_{R, T}^{\theta} & =-405640+5 T \\
\frac{1}{2} \mathrm{Ta}_{2} \mathrm{O}_{5}+\frac{11}{3} \mathrm{~B} & \rightarrow \mathrm{TaB}_{2}+\frac{5}{6} \mathrm{~B}_{2} \mathrm{O}_{3}(\mathrm{l}) \\
\Delta G_{R, T}^{\theta} & =-240372+2 T \\
\mathrm{WO}_{3}+4 \mathrm{~B} & \rightarrow \mathrm{WB}_{2}+\mathrm{B}_{2} \mathrm{O}_{3}(\mathrm{l}) \\
\Delta G_{R, T}^{\theta}= & -672560-22 T
\end{aligned}
$$

$$
\begin{gathered}
\mathrm{MoO}_{3}+4 \mathrm{~B} \rightarrow \mathrm{MoB}_{2}+\mathrm{B}_{2} \mathrm{O}_{3}(\mathrm{l}) \\
\Delta G_{R, T}^{\theta}=-603740-17 T \\
\frac{1}{5} \mathrm{TaB}_{2}+\frac{1}{5} \mathrm{NbB}_{2}+\frac{1}{5} \mathrm{TiB}_{2}+\frac{1}{5} \mathrm{WB}_{2} \\
+\frac{1}{5} \mathrm{MoB}_{2} \rightarrow\left(\mathrm{Ta}_{0.2} \mathrm{Nb}_{0.2} \mathrm{Ti}_{0.2} \mathrm{~W}_{0.2} \mathrm{Mo}_{0.2}\right) \mathrm{B}_{2} \\
\Delta G_{R, T}=\Delta G_{R, T}^{M} \\
\frac{1}{5} \mathrm{TiO}_{2}+\frac{1}{10} \mathrm{Ta}_{2} \mathrm{O}_{5}+\frac{1}{10} \mathrm{Nb}_{2} \mathrm{O}_{5} \\
+\frac{1}{5} \mathrm{WO}_{3}+\frac{1}{5} \mathrm{MoO}_{3}+\frac{41}{15} \mathrm{~B} \rightarrow \\
\left(\mathrm{Ta}_{0.2} \mathrm{Nb}_{0.2} \mathrm{Ti}_{0.2} \mathrm{~W}_{0.2} \mathrm{Mo}_{0.2}\right) \mathrm{B}_{2}+\frac{13}{15} \mathrm{~B}_{2} \mathrm{O}_{3}(\mathrm{l}) \\
\Delta G_{R, T}^{\theta}=-419250-5 T+\Delta G_{R, T}^{M}
\end{gathered}
$$

where $\Delta G_{R, T}^{M}$ is the mixing Gibbs free energy of HEB-1. In this case, the HEB-1 is assumed to be an ideal Raoultian solution and thereby $\Delta G_{R, T}^{M}$ can be calculated by the following equation:

$$
\Delta G_{R, T}^{M}=-T \Delta S_{\text {mix }}
$$

where $\Delta S_{\text {mix }}$ is the mixing entropy of HEB-1, which can be defined as $[13,14]$ :

$$
\Delta S_{\text {mix }}=-\frac{R}{3} \sum_{i=1}^{N} x_{i} \operatorname{In} x_{i}
$$

where $R$ is the ideal gas constant, $N$ is the metal element species, and $x_{i}$ are molar fractions of the $i$ th metal element in sublattices. According to Eq. (10), the $\Delta S_{\text {mix }}$ of HEB-1 is calculated to be about $0.54 R$. As a result, the $\Delta G_{R, T}^{\theta}$ of the reactions (7) and (8) can be mathematically expressed as

$$
\begin{gathered}
\Delta G_{R, T}^{\theta}=-5 T \\
\Delta G_{R, T}^{\theta}=-419250-10 T
\end{gathered}
$$

In such a case, the thermodynamics analysis of those possible chemical reactions for starting materials is depicted in Fig. 1(a). It can be observed that the standard Gibbs free energy of all reactions ((2)-(8)) is negative $\left(\Delta G_{R, T}^{\theta}<0\right)$, and therefore they can all proceed spontaneously. Nevertheless, it is worth noticing that the reaction (7) is very difficult to occur when it competes with other reactions due to its Gibbs free energy close to zero. But the reaction (8) is still prone to occur to generate HEB-1 products in the system owing to its very negative Gibbs free energy. That is to say, the 
synthesis of HEB-1 products via the reaction (8) using molten salt synthesis method is possible from the thermodynamic aspect. On the basis of the aforementioned theoretical analysis, we preformed the molten salt synthesis of HEB-1 products at different temperatures, and XRD characterization was conducted to determine phase compositions of the as-synthesized products at different temperatures. In order to observe the weak diffraction peaks more clearly, X-ray diffraction data was plotted on a logarithmic scale, as displayed in Fig. 1(b). Clearly, the as-synthesized products at $1273 \mathrm{~K}$ are composed of a dominant (Ta,Nb,Ti,W,Mo)B $\mathrm{B}_{2}$ phase and a minor secondary phase. According to JCPDS cards of NbB phase (JCPDS card No. 81-0911) and TaB phase (JCPDS card No. 81-0912), the minor secondary phase can be indexed to be ( $\mathrm{Ta}, \mathrm{Nb}) \mathrm{B}$ phase that possesses different crystal structure with major high-entropy diboride phase. With increase of the synthesis temperatures, diffraction of minor ( $\mathrm{Ta}, \mathrm{Nb}) \mathrm{B}$ phase gradually decreased and finally disappeared at $1423 \mathrm{~K}$ to form a single $\left(\mathrm{Ta}_{0.2} \mathrm{Nb}_{0.2} \mathrm{Ti}_{0.2} \mathrm{~W}_{0.2} \mathrm{Mo}_{0.2}\right) \mathrm{B}_{2}$ phase with a hexagonal crystal structure of metal diborides. Therefore, the pure
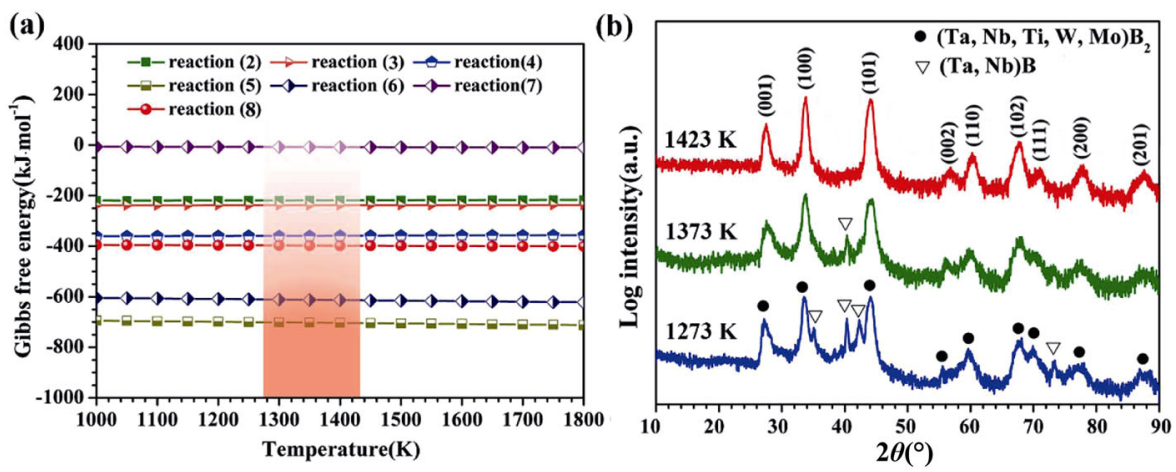

Fig. 1 (a) Thermodynamics analysis of the possible chemical reactions for the as-synthesized HEB-1 products; (b) XRD patterns of the as-synthesized products at different temperatures.

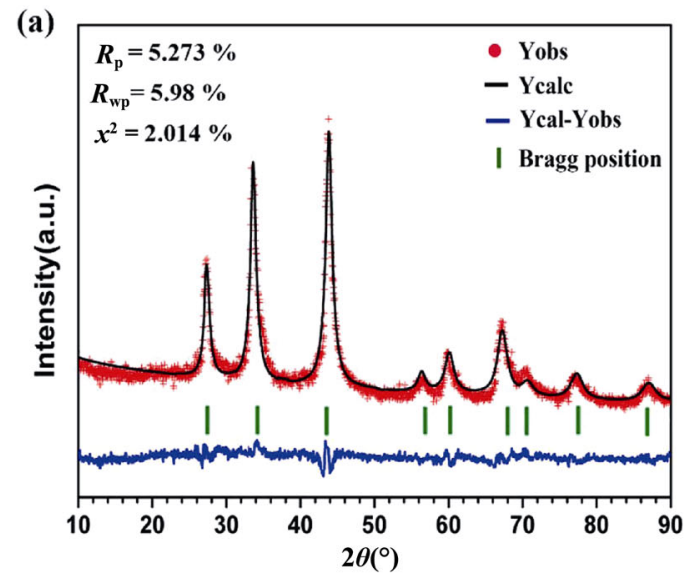

(b)

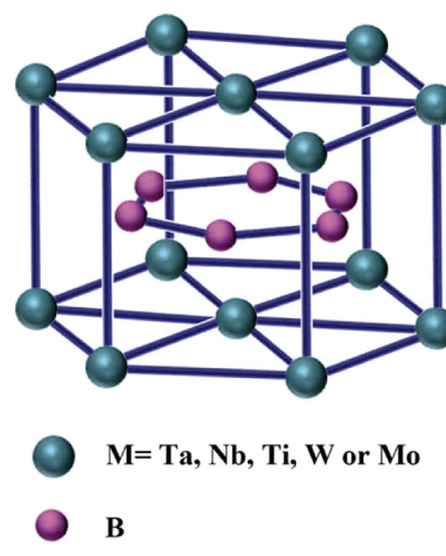

Fig. 2 (a) XRD pattern along with Rietveld refinement for the as-synthesized HEB-1 products at $1423 \mathrm{~K}$; (b) the crystal structure of the as-synthesized HEB-1 products. 
Table 1 Unit cell parameters and bond lengths of the as-synthesized HEB-1 products at $1423 \mathrm{~K}$ by XRD refinement and five individual diborides and the "average" values represent the means of five individual diborides

\begin{tabular}{ccccc}
\hline Sample & $a(\AA)$ & $c(\AA)$ & $\mathrm{B}-\mathrm{B}(\AA)$ & $\mathrm{M}-\mathrm{B}(\AA)$ \\
\hline $\mathrm{TaB}_{2}[24]$ & 3.076 & 3.265 & 1.811 & 2.467 \\
$\mathrm{NbB}_{2}[24]$ & 3.112 & 3.274 & 1.794 & 2.443 \\
$\mathrm{TiB}_{2}[24]$ & 3.028 & 3.228 & 1.745 & 2.357 \\
$\mathrm{WB}_{2}[23]$ & 3.023 & 3.057 & 1.741 & 2.356 \\
$\mathrm{MoB}_{2}[23]$ & 3.005 & 3.173 & 1.733 & 2.370 \\
Average & 3.048 & 3.199 & 1.764 & 2.398 \\
HEB-1 & 3.072 & 3.195 & 1.796 & 2.387 \\
\hline
\end{tabular}

Figure 3(a) presents low-magnification SEM image of the as-synthesized HEB-1 products. It can be observed that the as-synthesized HEB-1 products exhibit an interesting chrysanthemum-like morphology assembled from numerous nanorods. High-magnification SEM image (Fig. 3(b)) shows that each individual chrysanthemum-like structure is composed of several dozen well-aligned nanorods. Those nanorods possess a low aspect ratio with diameters of $20-30 \mathrm{~nm}$ and lengths of 100-200 nm. Figure 3(c) displays EDS compositional maps of the as-synthesized HEB-1 nanoflowers (labeled by a dotted green square in Fig. 3(a)) at micrometer scale. The distribution of five metal elements is highly homogeneous at micrometer scale and no segregation or aggregation is found throughout the scanned area, which implies that the as-synthesized HEB-1 nanoflowers possess the highly compositional uniformity at micrometer scale.

Figure 4(a) is a typical TEM image of an individual HEB-1 nanoflower, which clearly displays that the as-synthesized nanoflowers are composed of several dozen well-aligned nanorods with diameters of $20-30$ $\mathrm{nm}$ and lengths of 100-200 $\mathrm{nm}$ on a nanocluster root. The high-resolution transmission electron microscopy (HRTEM) images (Figs. 4(b) and 4(c)) of two selected nanorods in Fig. 4(a) show that these nanorods possess a periodic lattice structure with two sets of fringes with a $d$-space of $0.205 \mathrm{~nm}$ and $0.113 \mathrm{~nm}$ in Fig. 4(b) or $0.326 \mathrm{~nm}$ and $0.268 \mathrm{~nm}$ in Fig. 4(c), corresponding to $\{101\}$ and $\{2(-) 12\}$ planes or $\{001\}$ and $\{1(-) 10\}$ planes of $\left(\mathrm{Ta}_{0.2} \mathrm{Nb}_{0.2} \mathrm{Ti}_{0.2} \mathrm{~W}_{0.2} \mathrm{Mo}_{0.2}\right) \mathrm{B}_{2}$, respectively. The corresponding fast Fourier transform (FFT) patterns (inserted in figs. 4(b) and 4(c)) display that those nanorods are single-crystal hexagonal structure of $\left(\mathrm{Ta}_{0.2} \mathrm{Nb}_{0.2} \mathrm{Ti}_{0.2} \mathrm{~W}_{0.2} \mathrm{Mo}_{0.2}\right) \mathrm{B}_{2}$. The HRTEM images, together with the corresponding FFT patterns, strongly demonstrate that these nanorods are single-crystal hexagonal structure of $\left(\mathrm{Ta}_{0.2} \mathrm{Nb}_{0.2} \mathrm{Ti}_{0.2} \mathrm{~W}_{0.2} \mathrm{Mo}_{0.2}\right) \mathrm{B}_{2}$. Meanwhile, the growth directions of nanorods presented in Figs. 4(b) and 4(c) can be determined as $<112>$ and $<001>$, respectively, which suggests that these nanorods grow up along the different directions. In addition, an amorphous layer of 2-3 nm can be observed on the surface of the nanorods, as shown in Figs. 4(b) and 4(c). Figure 4(d) shows the scanning transmission electron microscopy (STEM) image and the corresponding EDS compositional maps of two individual nanorods at nanometer scale. It is evident that the distribution of five metal elements in those nanorods is highly uniform at nanoscale without segregation or aggregation phenomenon and simultaneously five metal elements are in near equiatomic amounts (Table 2). In addition, a small amount of $\mathrm{O}$ element can be detected by EDS, as listed in Table 2, which can account for the presence of the amorphous $\mathrm{B}_{2} \mathrm{O}_{3}$ layer on the nanorod surface.

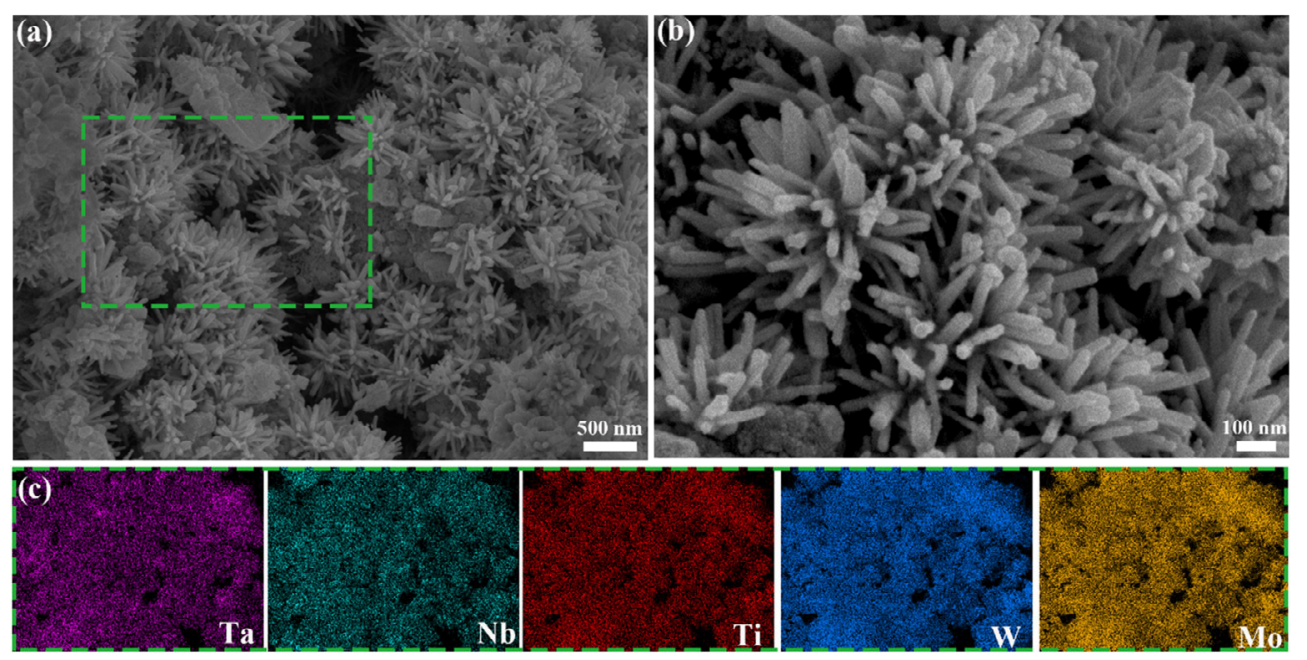

Fig. 3 SEM characterizations of the as-synthesized HEB-1 products: (a) low-magnification SEM image; (b) high-magnification SEM image; (c) EDS elemental maps. 


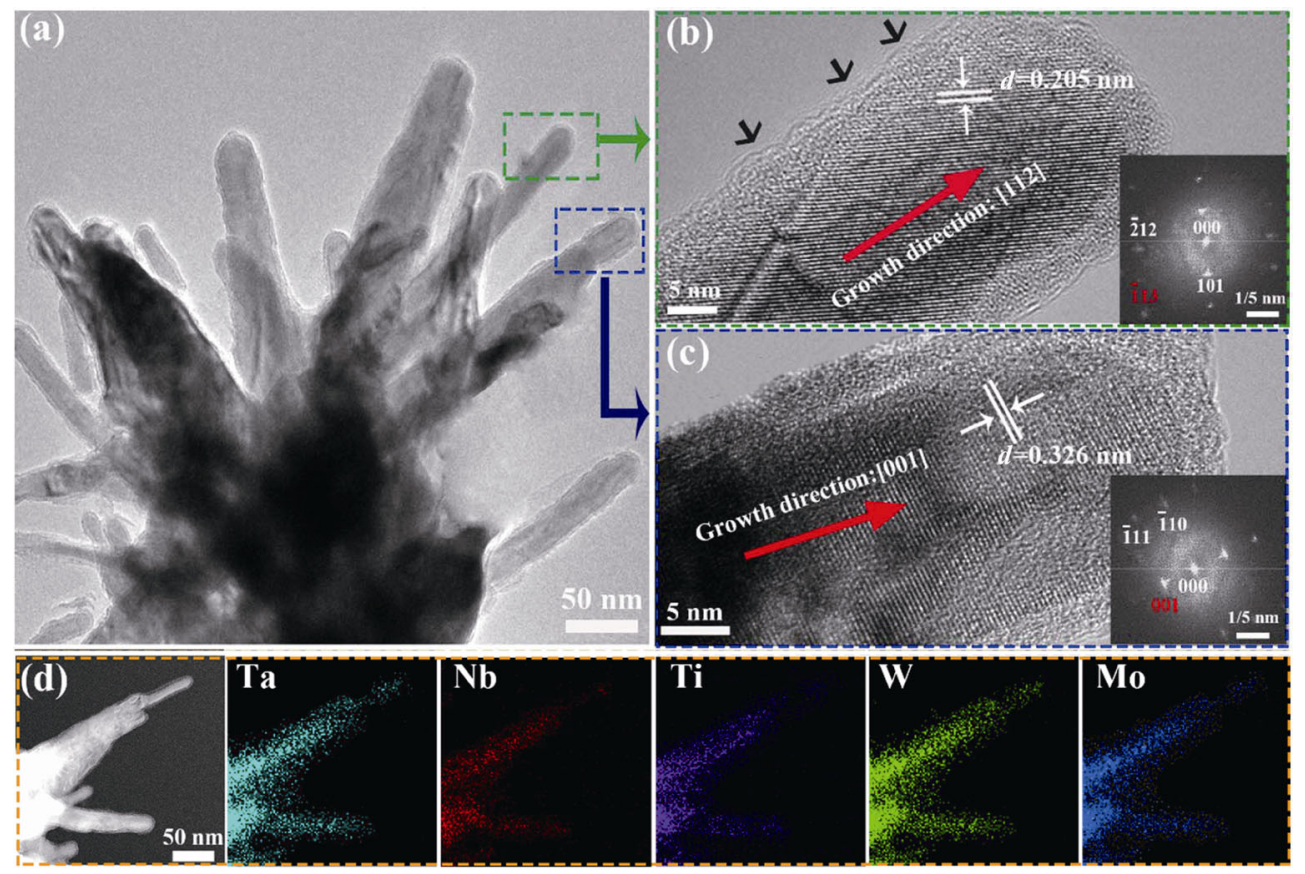

Fig. 4 TEM analyses of an individual HEB-1 nanoflower: (a) TEM image; (b) HRTEM image of the selected nanorod labeled by doted green square in (a); (c) HRTEM image of the selected nanorod labeled by doted blue square in (a); (d) STEM image and the corresponding EDS compositional maps.

Table 2 Elemental atomic percentage of the as-synthesized HEB-1 nanoflowers by STEM-EDS analyses

\begin{tabular}{cccccccc}
\hline Element & $\mathrm{Ti}$ & $\mathrm{W}$ & $\mathrm{Ta}$ & $\mathrm{Nb}$ & $\mathrm{Mo}$ & $\mathrm{B}$ & $\mathrm{O}$ \\
\hline$(\mathrm{at} \%)$ & 6.13 & 6.02 & 5.77 & 5.63 & 5.50 & 69.94 & 1.01 \\
\hline
\end{tabular}

In order to investigate the growth mechanism of the as-synthesized HEB-1 nanoflowers, the additional experiments were conducted at $1423 \mathrm{~K}$ for different holding time (10, 20, and $30 \mathrm{~min})$. XRD patterns along with Rietvelt refinement by using $P / 6 \mathrm{mmm}$ a hexagonal structure and SEM images of the as-synthesized products were displayed in Fig. 5. As presented in Figs. 5(a), 5(b), and 5(c), all the as-synthesized products possessed the same crystal structure and no other phases were observed. Meanwhile, it can be observed from Table 3 that the lattice parameters and bond lengths of these three products are in near equal, in good agreement with those of the as-synthesized HEB-1 nanoflowers (Table 1), which further confirms that they are all the same crystal structure and no other phases. However, these three products exhibited the different morphology. When the holding time was 10 min, the as-synthesized products showed many clusters with diameter of several hundred nanometers involving numerous spherical nanoparticles, as shown in Fig. 5(d). With the holding time increasing to $20 \mathrm{~min}$, numerous nanorods with diameters of $20-30 \mathrm{~nm}$ and lengths of 50-150 nm grew on formed cluster surface, as displayed in Fig. 5(e). As the holding time increased to $30 \mathrm{~min}$, the as-synthesized products possessed the chrysanthemum-like morphology assembled from numerous well-aligned nanorods, as presented in Fig. 5(f). These results can well demonstrate the growth process of the as-synthesized HEB-1 nanoflowers. In other words, the formation of the as-synthesized HEB-1 nanoflowers underwent a transformation from nanoclusters to nanoflowers. To further investigate the chemical composition of the different products during the nanoflower growth process, these three samples were analyzed by EDS and the results are shown in Figs. 5(d) $-5(\mathrm{~g})$. It can be found that all the samples have the same chemical composition and they consist of B element and five metal elements in near equiatomic amounts, as displayed in Fig. 5(g). What's more, EDS results show that there is no difference in chemical composition between roots and nanorods.

Table 3 Unit cell parameters and bond lengths of the as-synthesized HEB-1 products at $1423 \mathrm{~K}$ for different holding time by XRD refinement

\begin{tabular}{ccccc}
\hline Sample & $a(\AA)$ & $c(\AA)$ & $\mathrm{B}-\mathrm{B}(\AA)$ & $\mathrm{M}-\mathrm{B}(\AA)$ \\
\hline $10 \mathrm{~min}$ & 3.067 & 3.190 & 1.788 & 2.374 \\
$20 \mathrm{~min}$ & 3.082 & 3.214 & 1.803 & 2.417 \\
$30 \mathrm{~min}$ & 3.074 & 3.197 & 1.792 & 2.395 \\
\hline
\end{tabular}



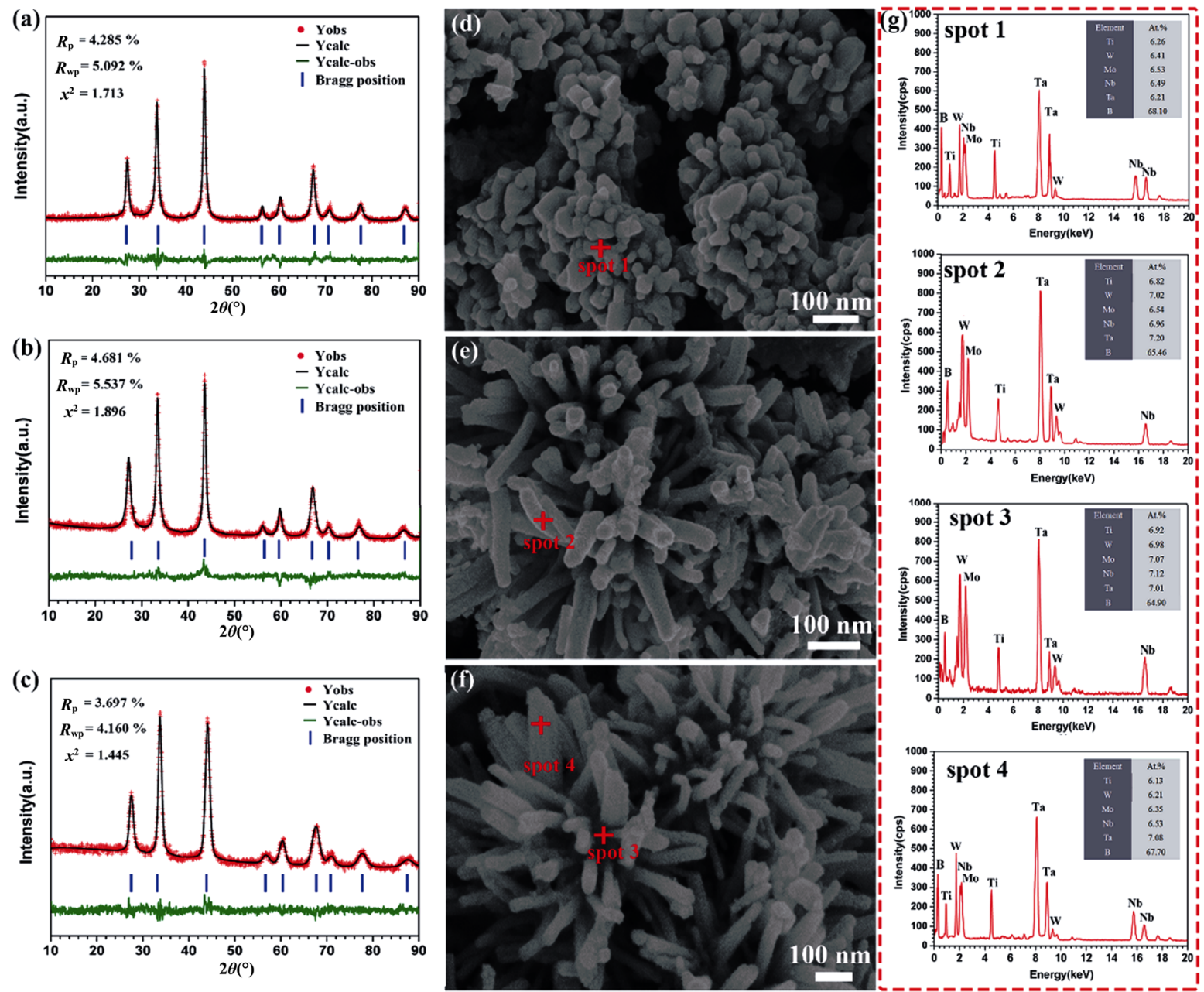

Fig. $5 \mathrm{XRD}$ and SEM characterizations of the as-synthesized products at $1423 \mathrm{~K}$ for the different holding time: (a) XRD pattern of 10 min along with Rietveld refinement; (b) XRD pattern of 20 min along with Rietveld refinement; (c) XRD pattern of 30 min along with Rietveld refinement; (d) SEM image of $10 \mathrm{~min}$; (e) SEM image of $20 \mathrm{~min}$; (f) SEM image of $30 \mathrm{~min}$, (g) EDS analyses of the as-synthesized samples in (d-f).

On the basis of the above-mentioned XRD, SEM, EDS, and TEM analyses, a possible growth process of the as-synthesized HEB-1 nanoflowers was proposed and the corresponding schematic diagram was depicted in Fig. 6. In our work, this system consisted of five metal oxides, B powders, and $\mathrm{KCl}$ salts, as presented in Fig. 6(a). At elevated temperature, $\mathrm{KCl}$ salts would first melt into liquid phase as molten salt medium and then five metal oxides $\left(\mathrm{MeO}_{x}\right)$ dissociated to mobile metal cations $\left(\mathrm{Me}^{x+}\right)$ and $\mathrm{O}^{2-}$ in the molten salt medium by the following equation [25]:

$$
\mathrm{MeO}_{x}=\mathrm{Me}^{x+}+\mathrm{O}^{2-}
$$

Meanwhile, B powders would also dissolve into the molten salt medium to form ions of $\mathrm{B}^{3+}$ and electrons as the following equation [25], as presented in Fig. 6(b).

$$
\mathrm{B}=\mathrm{B}^{3+}+3 \mathrm{e}^{-}
$$

Nevertheless, the solubility of $\mathrm{B}$ is very low in $\mathrm{KCl}$ molten salt medium, which is less than that of metal oxides in $\mathrm{KCl}$ molten salt medium [25]. In this situation, metal cations and $\mathrm{O}^{2-}$ would rapidly diffuse to the surface of $\mathrm{B}$ cations and reacted with each other to generate HEB-1 molecules and $\mathrm{B}_{2} \mathrm{O}_{3}$ by-products via the reaction (15), as illustrated in Fig. 6(c).

$$
\mathrm{Me}^{x+}+3 \mathrm{O}^{2-}+4 \mathrm{~B}^{3+}+\mathrm{e}^{-}=\mathrm{MeB}_{2}+\mathrm{B}_{2} \mathrm{O}_{3}
$$

where $\mathrm{MeB}_{2}$ presents HEB-1. This further confirmed the thermodynamics analyses of chemical reactions for the as-synthesized HEB-1 products. With the prolongation of the reaction time, the concentration of the generated HEB-1 molecules in molten salt medium would reach the supersaturation condition. Under this circumstances, the nucleation of HEB-1 would occur to generate numerous HEB-1 nucleus. Afterwards those nucleus would grow up to be the nanoflowers. In the following 
growth process, the formation of HEB-1 nanoflowers could be well interpreted by a classical interfacecontrolled growth instead of diffusion-controlled growth in molten salt medium based on Ostwald Ripening theory [26,27]. In general, the interfacecontrolled growth of crystal in solutions could be classified into two broad categories: rough interfacecontrolled growth and smooth surface-controlled growth [28]. Rough interface-controlled growth mechanism is generally utilized to interpret the growths of regular or spherical crystals, in which molecules can attach to the crystal surface at essentially any site to allow the interface to advance uniformly. While the smooth surface-controlled growth, which involves that molecules can only attach at the step to allow the growth to occur at a particular place, is usually used to explain the growths of a variety of oriented and hierarchical crystals. Whether the rough or smooth interface-controlled growth models can occur mainly depends on the intrinsic structure of materials and growth conditions. The Jackson $\alpha$-factor is usually proposed to predict whether the rough or smooth interface-controlled growth models can occur from the aspect of material's structure and it can be expressed as the following equation [29]:

$$
\alpha=\frac{\Delta H}{R T} \frac{\eta_{1}}{Z}
$$

where $\Delta H$ is enthalpy changes, $\eta_{1} / Z$ is the fraction of the nearest neighbor sites which are in a single layer of molecules at the surface, $T$ is the temperature, and $R$ is the gas constant. This growth model of crystal in solutions cannot be governed by the smooth interfacecontrolled growth mechanism unless the $\alpha$ value of materials is greater than 2 and simultaneously the supersaturation of the system is under a moderate or low condition [30]. For metal diborides, the $\alpha$ values of main planes, such as $\{001\},\{100\}$, and $\{110\}$, are greater than 4 [31], from which it can be inferred that the $\alpha$ values of planes for high-entropy metal diborides are also greater than 4 . As a result, these two surfacecontrolled growth models of crystal in solutions mainly depend on the growth conditions of the system. At an initial stage, the concentration of HEB-1 molecules in molten salt medium is expected to be constant under a high supersaturation condition. Such condition will favor the rough interface-controlled crystal growth. In other words, the formed HEB-1 nucleus in molten salt medium will undergo the rough interface-controlled growth to form individual nanoparticles with spherical morphology [32,33], as displayed in Fig. 6(c). Those individual nanoparticles are not stable in molten salt medium due to their high specific surface energy and they will be aggregated by strong van der Waals interaction among them to form nanoparticle clusters [34], as shown in Fig. 6(d). With the further prolongation of the reaction time, the concentration of HEB-1 molecules in molten salt medium will decrease to a moderate or low supersaturation condition. In this situation, the following growth of the nanoparticle clusters will be governed by the smooth interface-controlled growth. It is well known that the smooth interface-controlled growth includes two-dimensional (2D) nucleation growth and screw dislocation growth [35]. According to SEM and TEM observations, the following growth of the nanoparticle clusters should be governed by the smooth interface-controlled 2D nucleation growth. According to the 2D nucleation growth theory, the growth rate $\left(v_{\mathrm{c}}\right)$ at the nucleation plane can expressed as [36]:

$$
v_{\mathrm{c}}=a v \sigma \cdot \exp \left(\frac{E_{\mathrm{des}}-E_{\mathrm{sd}}}{2 k T}\right) \exp \left(-\frac{\Delta U}{k T}\right)
$$

where $a$ is the distance between adsorption sites, $v$ is the vibration frequency, $\sigma$ is the supersaturation, $k$ is the Boltzmann constant, $T$ is the temperature, $\Delta U$ is the active energy, $E_{\text {des }}$ is the activation energy for the desorption of an admolecule from the crystal surface, and $E_{\mathrm{sd}}$ is the activation energy for surface diffusion. For high-entropy metal diborides, the $\{100\}$ plane possessed the highest surface desorption energy $\left(E_{\text {des }}\right)$ among all crystal facets [37]. Consequently, the growth velocity of the $<100>$ direction should be the highest among all directions in thermodynamics. However, in a realistic crystal growth process, some environmental factors of the system, such as impurity pinning sites, adsorbed surfactants, and transport kinetics, are complicated and varied, which can also result in the highest growth velocity in other directions in addition to $<100>$ direction [38]. This is why the growth directions of the nanorods in HEB-1 nanoflowers are different, as displayed in Figs. 4(b) and 4(c). In brief, under a moderate or low supersaturation condition, HEB-1 molecules in molten salt medium will nucleate on the surface active positions of the formed nanoclusters to decrease the system energy and simultaneously they will grow along the direction of the lowest energy based on the smooth interface-controlled 2D nucleation growth. Finally, the complicated and varied environmental factors resulted in the formation of HEB-1 nanoflowers with nanocluster roots based on the smooth interfacecontrolled 2D nucleation growth, as displayed in Fig. 6(f). 
(a)

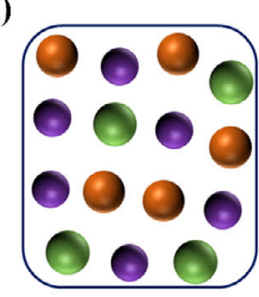

(f)

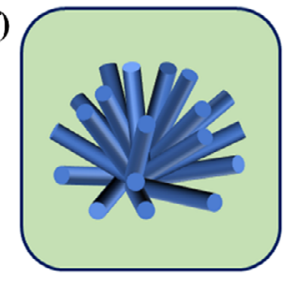

- $\mathrm{Me}^{4+}$

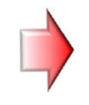

(b)

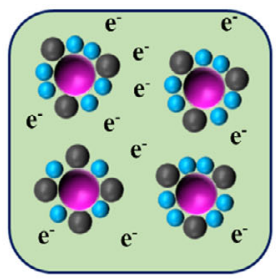

(e)

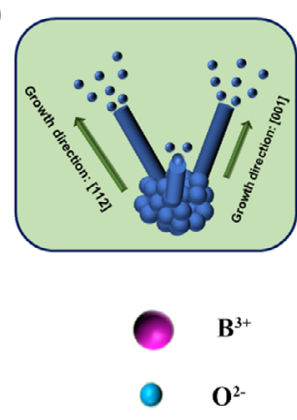

(c)

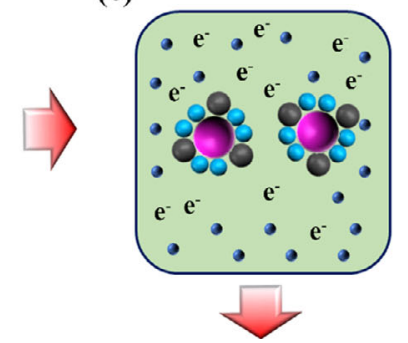

(d)

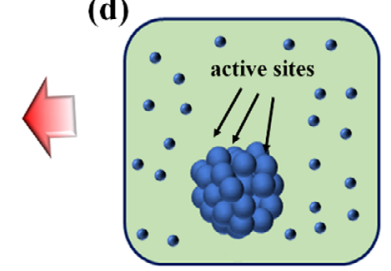

$\mathrm{KCl}$

$(\mathrm{Ta}, \mathrm{Nb}, \mathrm{Ti}, \mathrm{W}, \mathrm{Mo}) \mathrm{B}_{2}$

Fig. 6 Schematic diagram of the possible growth process for HEB-1 nanoflowers.

\section{Conclusions}

In summary, a new class of high-entropy nanomaterials, namely HEB-1 nanoflowers, was reported in our work for the first time. We first theoretically analyzed the formation possibility of HEB-1 from two aspects of lattice size difference and chemical reaction thermodynamics. The HEB-1 nanoflowers then were successfully synthesized by a facile molten salt synthesis method. The as-synthesized HEB-1 nanoflowers exhibited a chrysanthemum-like morphology assembled from numerous well-aligned nanorods with diameters of 20-30 nm and lengths of 100-200 nm. These nanorods had a single-crystalline hexagonal structure of metal diborides and simultaneously exhibited highly compositional uniformity from nanoscale to microscale. On the basis of SEM and TEM analyses, a classical surface-controlled crystal growth theory was proposed to interpret the formation of the as-synthesized HEB-1 nanoflowers.

\section{Acknowledgements}

We acknowledge financial support from the National Key R\&D Program of China (No. 2017YFB0703200), National Natural Science Foundation of China (Nos. 51802100 and 51972116), Young Elite Scientists Sponsorship Program by CAST (No. 2017QNRC001), and Guangdong Basic and Applied Basic Research Foundation (No. 2019A1515012145).

\section{References}

[1] Tsai MH, Yeh JW. High-entropy alloys: A critical review. Mater Res Lett 2014, 2: 107-123.

[2] Rost CM, Sachet E, Borman T, et al. Entropy-stabilized oxides. Nat Commun 2015, 6: 8485.

[3] Gludovatz B, Hohenwarter A, Catoor D, et al. A fractureresistant high-entropy alloy for cryogenic applications. Science 2014, 345: 1153-1158.

[4] Lei ZF, Liu XJ, Wu Y, et al. Enhanced strength and ductility in a high-entropy alloy via ordered oxygen complexes. Nature 2018, 563: 546-550.

[5] Li ZM, Pradeep KG, Deng Y, et al. Metastable high-entropy dual-phase alloys overcome the strength-ductility trade-off. Nature 2016, 534: 227-230.

[6] Hong WC, Chen F, Shen Q, et al. Microstructural evolution and mechanical properties of $(\mathrm{Mg}, \mathrm{Co}, \mathrm{Ni}, \mathrm{Cu}, \mathrm{Zn}) \mathrm{O}$ highentropy ceramics. J Am Ceram Soc 2019, 102: 2228-2237.

[7] Li F, Zhou L, Liu JX, et al. High-entropy pyrochlores with low thermal conductivity for thermal barrier coating materials. $J$ Adv Ceram 2019, 8: 576-582.

[8] Ye BL, Wen TQ, Huang KH, et al. First-principles study, fabrication, and characterization of $\left(\mathrm{Hf}_{0.2} \mathrm{Zr}_{0.2} \mathrm{Ta}_{0.2} \mathrm{Nb}_{0.2} \mathrm{Ti}_{0.2}\right) \mathrm{C}$ high-entropy ceramic. J Am Ceram Soc 2019, 102: 4344-4352.

[9] Yan XL, Constantin L, Lu YF, et al. $\left(\mathrm{Hf}_{0.2} \mathrm{Zr}_{0.2} \mathrm{Ta}_{0.2} \mathrm{Nb}_{0.2} \mathrm{Ti}_{0.2}\right) \mathrm{C}$ high-entropy ceramics with low thermal conductivity. $\mathrm{J} \mathrm{Am}$ Ceram Soc 2018, 101: 4486-4491.

[10] Harrington TJ, Gild J, Sarker P, et al. Phase stability and mechanical properties of novel high entropy transition metal carbides. Acta Mater 2019, 166: 271-280.

[11] Ye BL, Wen TQ, Nguyen MC, et al. First-principles study, fabrication and characterization of $\left(\mathrm{Zr}_{0.25} \mathrm{Nb}_{0.25} \mathrm{Ti}_{0.25} \mathrm{~V}_{0.25}\right) \mathrm{C}$ high-entropy ceramics. Acta Mater 2019, 170: 15-23.

[12] Feng L, Fahrenholtz WG, Hilmas GE. Low-temperature 
sintering of single-phase, high-entropy carbide ceramics. $J$ Am Ceram Soc 2019, 102: 7217-7224.

[13] Gild J, Zhang YY, Harrington T, et al. High-entropy metal diborides: A new class of high-entropy materials and a new type of ultrahigh temperature ceramics. Sci Rep 2016, 6: 37946.

[14] Liu D, Wen TQ, Ye BL, et al. Synthesis of superfine high-entropy metal diboride powders. Scripta Mater 2019, 167: $110-114$

[15] Chen H, Zhao Z, Xiang, H, et al. Effect of reaction routes on the porosity and permeability of porous high entropy $\left(\mathrm{Y}_{0.2} \mathrm{Yb}_{0.2} \mathrm{Sm}_{0.2} \mathrm{Nd}_{0.2} \mathrm{Eu}_{0.2}\right) \mathrm{B}_{6}$ for transpiration cooling. $J$ Mater Sci Technol 2020, 38: 80-85.

[16] Liu D, Liu HH, Ning SS, et al. Synthesis of high-purity high-entropy metal diboride powders by boro/carbothermal reduction. J Am Ceram Soc 2019, 102: 7071-7076.

[17] Xia Y, Yang P, Sun Y, et al. One-dimensional nanostructures: Synthesis, characterization, and applications. Adv Mater 2003, 15: 353-389.

[18] Chu YH, Jing SY, Yu X, et al. High-temperature plateau-Rayleigh growth of beaded $\mathrm{SiC} / \mathrm{SiO}_{2}$ nanochain heterostructures. Cryst Growth Des 2018, 18: 2941-2947.

[19] Yao YG, Huang ZN, Xie PF, et al. Carbothermal shock synthesis of high-entropy-alloy nanoparticles. Science 2018, 359: 1489-1494.

[20] Ning SS, Wen TQ, Ye BL, et al. Low-temperature molten salt synthesis of high-entropy carbide nanopowders. $\mathrm{J} \mathrm{Am}$ Ceram Soc 2020, 103: 2244-2251.

[21] Djenadic R, Sarkar A, Clemens O, et al. Multicomponent equiatomic rare earth oxides. Mater Res Lett 2017, 5: 102-109.

[22] Kharisov B. A review for synthesis of nanoflowers. Recent Patents Nanotechnol 2008, 2: 190-200.

[23] Li P, Ma LS, Peng MJ, et al. Elastic anisotropies and thermal conductivities of $\mathrm{WB}_{2}$ diborides in different crystal structures: A first-principles calculation. J Alloys Compd 2018, 747: 905-915.

[24] Fahrenholtz WG, Wuchina EJ, Lee WE, et al. Ultra-high Temperature Ceramics: Materials for Extreme Environment Applications. John Wiley \& Sons, Inc., 2014.

[25] Kaptay G, Kuznetsov SA. Electrochemical synthesis of refractory borides from molten salts. Plasmas Ions 1999, 2: 45-56.

[26] Kirkpatrick RJ. Crystal growth from the melt: A review. American Mineralogist 1975, 60: 798-814.

[27] Ardell AJ, Ozolins V. Trans-interface diffusion-controlled coarsening. Nat Mater 2005, 4: 309-316.
[28] Jackson KA. The interface kinetics of crystal growth processes. Interface Sci 2002, 10: 159-169.

[29] Elwenspoek M. Comment on the $\alpha$-factor of Jackson for crystal growth from solution. J Cryst Growth 1986, 78: 353-356.

[30] Wagner RS, Ellis WC. Vapor-liquid-solid mechanism of single crystal growth. Appl Phys Lett 1964, 4: 89-90.

[31] Shiohara Y, Endo A. Crystal growth of bulk high-Tc superconducting oxide materials. Mater Sci Eng: R: Rep 1997, 19: 1-86.

[32] Kang MK, Kim DY, Hwang NM. Ostwald ripening kinetics of angular grains dispersed in a liquid phase by twodimensional nucleation and abnormal grain growth. $J$ Eur Ceram Soc 2002, 22: 603-612.

[33] Jo W, Kim DY, Hwang NM. Effect of interface structure on the microstructural evolution of ceramics. J Am Ceram Soc 2006, 89: 2369-2380.

[34] Zhou WZ. Reversed crystal growth: Implications for crystal engineering. Adv Mater 2010, 22: 3086-3092.

[35] Sunagawa I. Crystals: Growth, Morphology, \& Perfection. Cambridge University Press, 2007.

[36] Vesselinov MI. Crystal Growth for Beginners: Fundamentals of Nucleation, Crystal Growth and Epitaxy. World Scientific, 2016.

[37] Sun W, Liu JC, Xiang HM, et al. A theoretical investigation on the anisotropic surface stability and oxygen adsorption behavior of $\mathrm{ZrB}_{2}$. J Am Ceram Soc 2016, 99: 4113-4120.

[38] Chu YH, Jing SY, Liu D, et al. Morphological control and kinetics in three dimensions for hierarchical nanostructures growth by screw dislocations. Acta Mater 2019, 162 284-291.

Open Access This article is licensed under a Creative Commons Attribution 4.0 International License, which permits use, sharing, adaptation, distribution and reproduction in any medium or format, as long as you give appropriate credit to the original author(s) and the source, provide a link to the Creative Commons licence, and indicate if changes were made.

The images or other third party material in this article are included in the article's Creative Commons licence, unless indicated otherwise in a credit line to the material. If material is not included in the article's Creative Commons licence and your intended use is not permitted by statutory regulation or exceeds the permitted use, you will need to obtain permission directly from the copyright holder.

To view a copy of this licence, visit http://creativecommons.org/licenses/by/4.0/. 